\title{
HOT CARRIER DYNAMICS IN THE X VALLEY IN Si AND Ge MEASURED BY PUMP-IR-PROBE ABSORPTION SPECTROSCOPY
}

\author{
W. B. Wang, M. A. Cavicchia, and R. R. Alfano \\ Institute for Ultrafast Spectroscopy and Lasers, and New York State Center \\ for Advanced Technology for Uitrafast Photonic Materials and Applications, \\ The City College of New York, Convent Avenue at 138th St, NY, NY 10031
}

$\mathrm{Si}$ is the semiconductor of choice for nanoelectronic roadmap into the next century for computer and other nanodevices. With growing interest in $\mathrm{Si}, \mathrm{Ge}$, and $\mathrm{Si}_{\mathrm{m}} \mathrm{Ge}_{\mathrm{n}}$ strained superlattices, knowledge of the carrier relaxation processes in these materials and structures has become increasingly important. ${ }^{1}$ The limited time resolution for earlier studies ${ }^{2,3}$ of carrier dynamics in $\mathrm{Ge}$ and $\mathrm{Si}$, performed using $\mathrm{N}_{\mathrm{d}}$ :glass lasers, was not sufficient to observe the fast cooling processes.

In this paper, we present a direct measurement of hot carrier dynamics in the satellite $X$ valley in Si and Ge by time-resolved infrared(IR) absorption spectroscopy, and show the potential of our technique to identify whether the $\mathrm{X}$ valley is the lowest conduction valley in semiconductor materials and structures.

The details of the femtosecond visible-pump and IR-probe absorption set up has been reported elsewhere ${ }^{4}$ In this measurement, $\sim 585 \mathrm{~nm}, \sim 400$ fs pulses, obtained from the output of a synchronously pumped dye laser with a pulse-dye-amplifier, was used as a pump, and IR pulses tuned from $2.5 \mu \mathrm{m}$ to $5.5 \mu \mathrm{m}$, generated in a $\mathrm{LiIO}_{3}$ crystal by difference frequency method, was used to monitor the photoinduced IR absorption.

The measured change of the induced IR absorption at $\lambda_{\text {probe }}=3.3 \mu \mathrm{m}$ as a function of delay time between pump and probe pulses for intrinsic $\mathrm{Si}$ and Ge samples at room temperature are displayed in Figs. 1 and 2, respectively. The salient features of the curves are the following: although the curve for $\mathrm{Si}$ is characterized by a long flat decay within the measured time range of $\sim 30 \mathrm{ps}$, the curve for Ge has a short decay of $\sim 6 \mathrm{ps}$ followed by a long flat decay.

The measurements were also extended to other probe wavelengths of $2.8 \mu \mathrm{m}, 4.5$ $\mu \mathrm{m}$ and $5.2 \mu \mathrm{m}$ for both Si and Ge. The time evolution of the induced IR absorption at those probe wavelengths was found to be similar as that for $\lambda_{\text {probe }}=3.3 \mu \mathrm{m}$ : a single flat decay appears with $\mathrm{Si}$, and a short decay followed by a flat decay accompanies with $\mathrm{Ge}$. It is clear that the different temporal behavior of the induced IR absorption for Si and Ge reflects their different carrier cooling processes and arises from their different band structures.

The induced total IR absorption is attributed to interconduction band absorption (ICA) by electrons from the lower $\mathrm{X}$ valley to the higher $\mathrm{X}$ valley separated by an energy difference in IR; free carrier absorption (FCA) by holes in the valence bands and by electrons in the conduction bands; and inter-valence band absorption (IVA) by electrons from the splitoff to the heavy and light hole bands. Since the calculated values ${ }^{3,5}$ of OD for IVA at the probe wavelengths are much smaller than the measured total OD, IVA can be neglected in our 


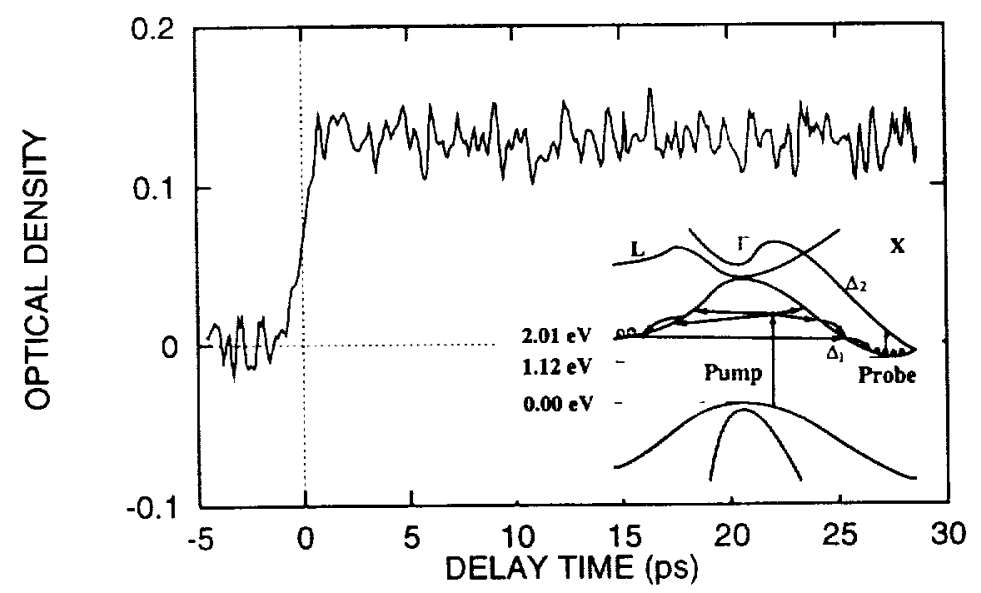

Figure 1. The measured change of the induced IR absorption as a function of delay time at $\lambda_{\text {probe }}=3.3 \mu \mathrm{m}$ in $\mathrm{Si}$. The energy band structure of $\mathrm{Si}$ is shown in the inset.

consideration. The decay of FCA is flat because the recombination times of electrons and holes in $\mathrm{Ge}$ and $\mathrm{Si}$ are several order of magnitude longer than our experimental time scale. The long flat decay of FCA has been previously observed. ${ }^{2-4}$

The temporal characteristics of ICA in Si and Ge depends on their energy band structures. In $\mathrm{Si}$, the lowest conduction band energy $(1.12 \mathrm{eV}$ measured from the top of the $\Gamma$ valence band) is located at the $X\left(\Delta_{1}, \Delta_{2}\right)$ valley ${ }^{6}$ as shown in the inset of Fig. 1 . Hot carriers produced by $585 \mathrm{~nm}$ pump photons through an indirect transition can obtain sufficient energy to reach both the $\mathrm{X}$ and $\mathrm{L}$ valleys, from which they can scatter to each other as well as scatter inside each of the $X$ and $L$ valleys. Since the energy of the $X$ valley minimum is lower than that of the $\mathrm{L}$ valley $(2.01 \mathrm{eV})^{7}$, after a few times of the $\mathrm{X}-\mathrm{L}$ backand-forward intervalley scattering, almost all of the electrons will finally scatter into the $\mathrm{X}$ valley. Those $X$ valley electrons then decay to the bottom of the $X$ valley through intravalley scattering, and stay there until they combine with holes in a few hundred nanoseconds. ${ }^{8}$ Therefore, the induced ICA by $X$ valley electrons from $\Delta_{1}$ to $\Delta_{2}$ (see the inset of Fig.1) has a long flat decay.

In contrast to $\mathrm{Si}$, the lowest conduction band energy in $\mathrm{Ge}$ is located at the $\mathrm{L}$ valley 6 $(0.66 \mathrm{eV})$ as shown in the inset of Fig.2. Electrons pumped by $585 \mathrm{~nm}$ photons through direct transition in the $\Gamma$ valley obtain enough energy to undergo intervalley scattering from the $\Gamma$ valley to the $L$ and $X$ valleys. The population of electrons in the $X$ valley increases at first to its maximum through the $\Gamma, \mathrm{L}->\mathrm{X}$ intervalley scattering. Since the minimum energy of the $X$ valley $(0.86 \mathrm{eV})$ is higher than that of the $\Gamma$ and $L$ valleys $(0.80 \mathrm{eV}$ and $0.66 \mathrm{eV}$, respectively), electrons stay in the $\mathrm{X}$ valley for only a short time, and then decay back to the $\Gamma$ and $L$ valleys. Therefore, the ICA in the $X$ valley should have a short decay.

In order to quantitatively understand the change of the ICA with band structures, a parameter $f_{X}$, defined as the fraction of carrier density in the $X$ valley relative to the total carrier density at the delay time corresponding to the flat absorption region, should be discussed. Since electrons in the conduction bands at the flat absorption region may be assumed to be in thermal equilibrium and characterized by a Boltzmann distribution, $\mathrm{f}_{\mathrm{X}}$ can be written ${ }^{9}$ as:

$$
\mathrm{f}_{\mathrm{X}}=1 /\left[1+\left(\mathrm{t}_{\mathrm{X}} / \mathrm{t}_{\mathrm{L}}\right)\left(\mathrm{m}_{\mathrm{L}} / \mathrm{m}_{\mathrm{X}}\right) 3 / 2 \exp \left(\Delta \mathrm{E}_{\mathrm{XL}} / \mathrm{K}_{\mathrm{B}} \mathrm{T}\right)+\left(\mathrm{t}_{\mathrm{X}} / \mathrm{t}_{\Gamma}\right)\left(\mathrm{m}_{\Gamma} / \mathrm{m}_{\mathrm{X}}\right) 3 / 2 \exp \left(\Delta \mathrm{E}_{\mathrm{X}} / \mathrm{K}_{\mathrm{B}} \mathrm{T}\right)\right]
$$

where $t_{i}$ and $m_{i}$ are the lifetime and density-of-state effective mass of electrons in the ith $(i=\Gamma, L$ and $X)$ conduction valley, respectively; $\Delta E_{x i}=\left(E_{g}\right)^{x}-\left(E_{g}\right)^{i}$ is energy difference between the $X$ and ith vallys ( $i=\Gamma, L$ ) at their minima; $K_{B}$ is the Boltzmann constant and $T$ is 


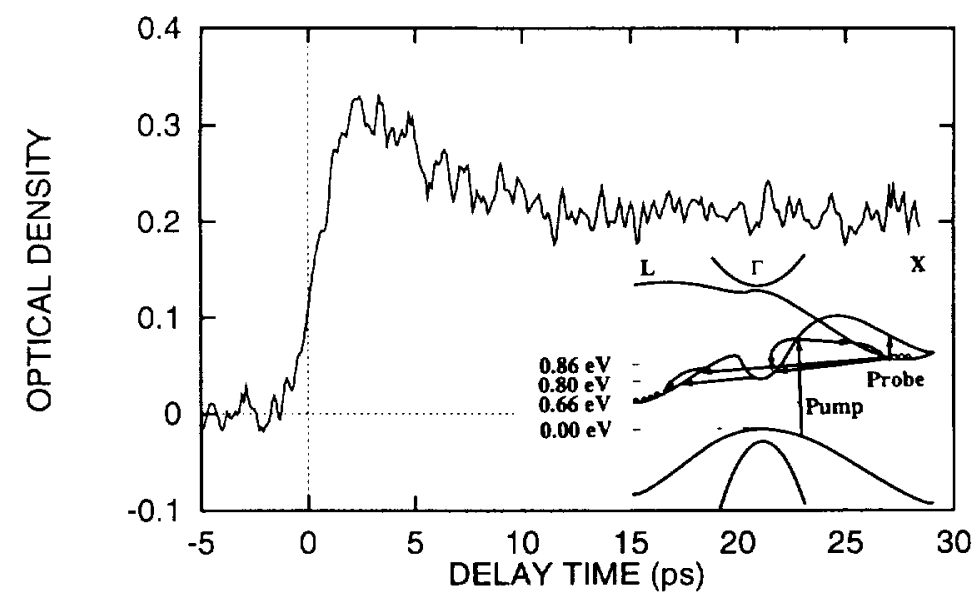

Figure 2. The measured change of the induced IR absorption as a function of delay time at $\lambda_{\text {probe }}=3.3 \mu \mathrm{m}$ in $\mathrm{Ge}$. The energy band structure of $\mathrm{Ge}$ is shown in the inset.

the effective temperature of relaxed electrons $\left(T=293 \mathrm{~K}\right.$ ). For $\mathrm{Si}$, substituting $\Delta \mathrm{E}_{\mathrm{XL}}=-890$ $\mathrm{meV}$ and other parameters ${ }^{6,8}$ of $\mathrm{t}_{\mathrm{X}}, \mathrm{t}_{\mathrm{L}}, \mathrm{mX}_{\mathrm{X}}$, and $\mathrm{m}_{\mathrm{L}}$ into eqn.(1), $\mathrm{f}_{\mathrm{X}}$ was calculated to be $\left[\mathrm{f}_{\mathrm{X}}\right]_{\mathrm{Si}} \sim 1$, which indicates that almost all of the electrons will decay and stay at the bottom of the $\mathrm{X}$ valley. Therefore, the ICA for $\mathrm{Si}$ is flat. For Ge, substituting values of $\triangle \mathrm{E}_{\mathrm{XL}}=200$ meV and $\Delta E_{X}=60 \mathrm{meV}$, and other parameters 6,8 of $t_{i}, m_{i},(i=L, \Gamma)$ into eqn. $(1), f_{X}$ was calculated to be $\left[f_{X}\right] G e \sim 0$, which means that the $X$ valley holds almost no electrons at the long delay time. Since electrons stay in the $\mathrm{X}$ valley only for a short time, the ICA for Ge has a short decay.

The temporal behavior of the photoinduced IR absorption in a semiconductor can be used as a criteria to determine whether the $X$ valley is the lowest conduction band valley. According to eqn.(1), materials with a conduction band energy minimum at the $X$ valley $\left(\Delta E_{X i}<0\right.$ for $i=\Gamma$ and $L$, therefore, $\left.f_{X} \sim 1\right)$ should have a single flat decay, while materials with a conduction band valley minimum not at the $X$ valley $\left(\Delta E_{X i}>0\right.$ for $i=\Gamma$ or $L$, therefore, $f_{X} \sim 0$ ) should have a short decay followed by a flat decay if the pump and probe wavelengths used can make the transition for ICA in the $\mathrm{X}$ valley. Over past several years, it has become a hot and controversial topic that certain $\mathrm{Si}_{m} \mathrm{Ge}_{\mathrm{n}}$ strained superlattices may be converted from the indirect band gap of Si to a direct band gap via Brillouin zone folding. ${ }^{1}$ The pump-IR-probe absorption technique presented here will be particularly useful for identifying this fundamental change of the lowest conduction band from the $\mathrm{X}$ valley to the $\Gamma$ valley.

This research is supported by NASA and the NYS Technology Foundation.

References

1. H.Presting, H.Kibbel, M.Jaros, R.M.Turton, U.Menczigar, G.Abstreiter, and H.G. Grinmeiss, Semicond. Sci. Technol., 7, 1127 (1992).

2. A.L.Smirl, J.Ryanlindle, and S.C.Moss, Phys. Rev., B18, 5489 (1978).

3. N.Ockman, R.Dorsinville, W.B.Wang and R.R.Alfano, IEEE QE-23, 2008 (1987).

4. W.B.Wang, K.Shum, R.R.Alfano, D.Szmyd, and A.J.Nozik, Phys. Rev. Lett., $\underline{68}$ 662 (1992).

5. R.Braustein, Phys. Rev., 130 869 (1963).

6. O.Madelung, in "Semiconductors: Group IV and III-V Compounds, in Data in Science and Technology", edited by R. Poerschke (Springer, Berlin, 1991).

7. J.Weber and M.I.Alonso, Phys. Rev., B40, 5683 (1989-I).

8. B.R.Nag, in "Semiconductors probed by ultrafast laser spectroscopy", edited by R. R. Alfano (Academic, Orlando, 1984), Vol.I, Ch. 1.

9. W.B.Wang, R.R.Alfano, D.Szmyd, and A.J.Nozik, Phys. Rev., B46, 15828 (1992). 


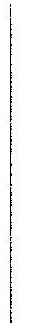

